\title{
MIXED HYBRID AND ELECTRIC BUS DYNAMIC FLEET MANAGEMENT IN URBAN NETWORKS: A MODEL PREDICTIVE CONTROL APPROACH
}

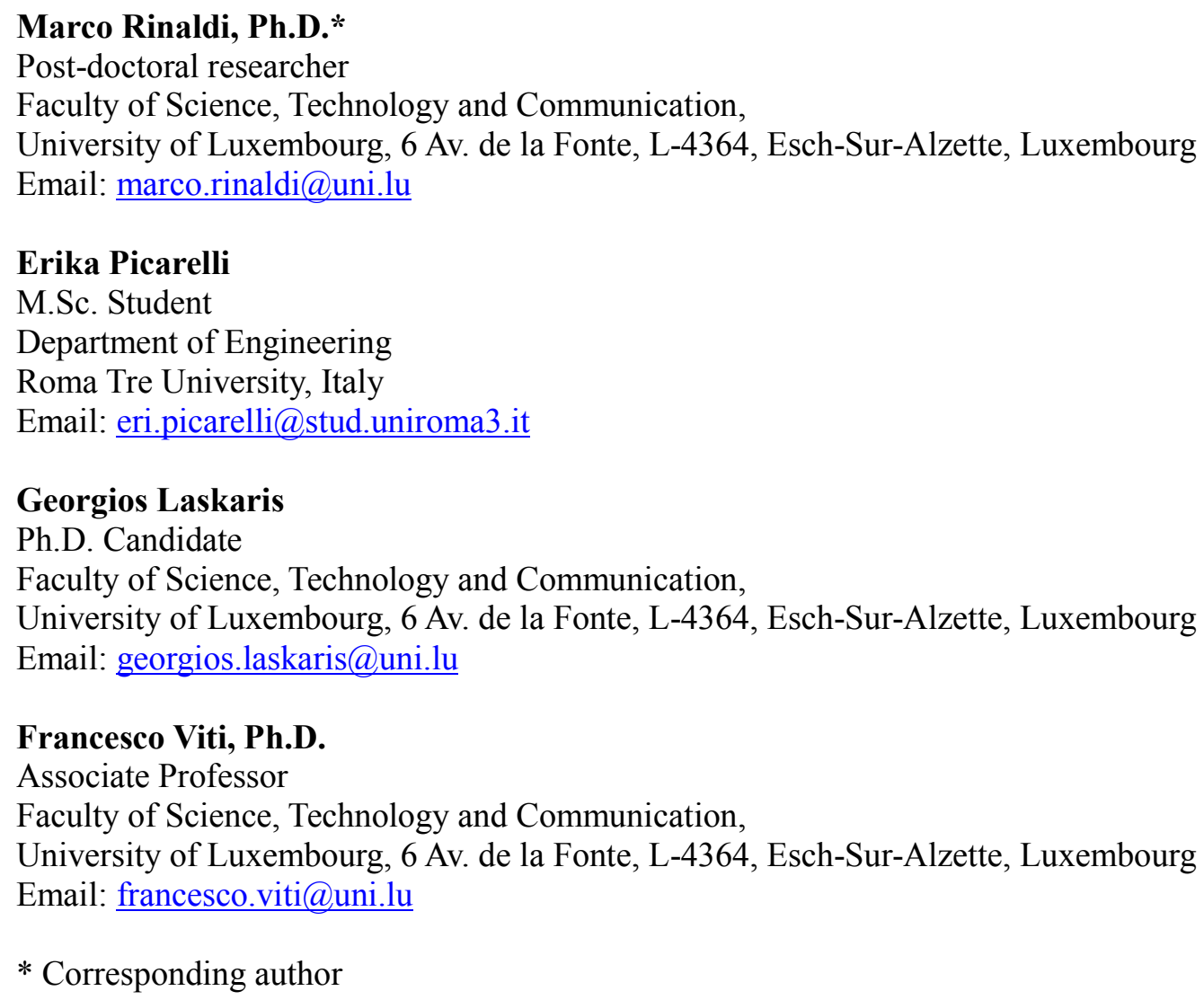

Word count: 5817 words text +2 tables x 250 words (each) $=6317$ words

Submission Date 15/11/2018 


\section{ABSTRACT}

3

4

Reducing pollutant emissions and promoting sustainable mobility solutions, including Public Transport, are increasingly becoming key objectives for policymakers worldwide. In order to jointly achieve these goals, careful consideration should be put on the operational cost and management of PT services, in order to promote the adoption of green mobility solutions and advanced management techniques by operators. In this work we develop a dynamic fleet management approach for next generation Public Transportation systems, considering the instance of mixed electric / hybrid fleet. Our objective is that of investigating to what extent electrification, coupled with optimal fleet management, can yield operational cost savings for PT operators, explicitly considering real-time disturbances, including delays, service disruptions etc. We propose a Mixed Integer Linear Program to address the problem of optimal scheduling of a mixed fleet of electric and hybrid / non-electric buses, and employ it as predictor in a Model Predictive Control approach. Test results based upon a real-life scenario showcase how the proposed approach is indeed capable of yielding a sizable reduction in operational costs, even when considerable disturbances arise from the underlying system.

Keywords: Dynamic bus fleet management, e-bus charging scheduling, MILP, MPC 
The last four decades have witnessed the rapid and widespread adoption of private transport, due to significant advancements in technology. While yielding considerable gains at the individual level, this has resulted in negative externalities, both in terms of network inefficiencies (ever-increasing congestion causing delays, productivity loss, ...) and in terms of pollutant emissions. To counteract these effects, developing advanced solutions for sustainable urban Public Transport (PT) is becoming of paramount importance for policymakers, practitioners and researchers worldwide. In order to become an attractive alternative to the comfort and flexibility offered by private transport solutions, next generation PT services must however meet very demanding criteria both in terms of service performance and quality (minimal delays, high frequency, high resilience, ...). Next to these ambitious performance goals, researchers and practitioners are also actively investigating whether the adoption of greener vehicles (hybrid electric and/or full electric buses) can yield both reduced pollutant emissions and operational costs, representing therefore a win-win situation for both policymakers and PT operators.

Adopting fully electric vehicles isn't however straightforward from the tactical/operational perspective: ebuses exhibit shorter driving ranges compared to hybrid and conventional combustion alternatives, requiring within-day recharging operations, which poses extra constraints to the state-of-the-art problem of transit scheduling (1). Solutions to this problem must be developed with care, as a clear trade-off exists between exploiting the available full-electric fleet to serve scheduled trips at a reduced operational cost (compared to either internal combustion or hybrid buses), and the cost of recharging operations. Indeed, while recent technological advancements have substantially reduced the time required to fully recharge an e-bus battery, this comes at a considerable cost in terms of energy prices, due to the required peak current.

In the Luxembourgish national context, recent policies adopted by the government are supporting the capital's main PT operators towards widespread electrification (2). By the end of 2018, all buses operated by Autobus Ville de Luxembourg are indeed expected to be plug-in hybrid electric. Furthermore, a small number of opportunity charging stations has been equipped in selected terminals around the city, to encourage further adoption of full electric vehicles in the coming ten-year period. In collaboration with the main national stakeholders, the University of Luxembourg is investigating the conditions under which this shift can be optimal, both from the strategic and tactical/operational perspective.

In this paper we develop models and algorithms to support this paradigm shift, specifically addressing two research questions which we believe of both theoretical and practical relevance:

1) The problem of optimal scheduling and dispatching of a mixed fleet of electric and plug-in hybrid vehicles, bearing different recharging requirements, has received little attention in literature;

2) In high frequency services operating in congestion-sensitive networks, operational disturbances such as delays, bunching etc. might arise; Model-based dynamic fleet management approaches might be useful to reduce loss of performance without resorting to deadheading.

In what follows we propose a mixed-integer linear programming formulation of the single-depot vehicle scheduling problem (SDVSP), which explicitly considers the additional constraints deriving from the inclusion of electric vehicles and their discharging/recharging dynamics. This formulation, based upon our recent work (3), is accompanied by a time-window decomposition solution scheme, which allows both to i) tackle large scale problems at a minor loss in optimality and ii) employ the model as predictor in a Model Predictive Control framework. By basing ourselves upon the real-life scenario of Luxembourg City, we showcase both how relevant appropriate modelling is when computing an offline daily plan for the dispatching of a mixed plug-in hybrid and electric fleet, and, most importantly, how robust the proposed model is when employed in real-time fleet management applications. 
The rest of the paper is organized as follows. The next section provides a literature review of fleet management operations, highlighting both the existing works dealing with electrification and those focusing on operational research driven approaches to solve the dispatching problem optimally. Later we discuss the methodological contribution of this paper, focusing on the proposed model and its solution schemes, both for offline and online purposes. Experimental results will be presented next, evaluating and comparing how different dispatching techniques perform in terms of operational costs, both in offline and online instances. Finally, some conclusions and pointers for future research are drawn.

\section{LITERATURE REVIEW}

Vehicle scheduling problems in the context of public transportation have been studied extensively as part of the "full operational planning process", as defined in (4). From a mathematical / operations research perspective, this problem is known as the Single/Multi-Depot Vehicle Scheduling Problem (SDVSP/ MDVSP), for which several algorithms, both exact and heuristic in nature, have been developed (5). Indeed, exhaustive literature reviews concerning different algorithmic approaches for these problems can be found $(6,7)$.

Concerning the expected benefits of PT electrification in the urban context, works such as (8-11) have investigated the problem both from a technological perspective and from an economic standpoint, highlighting the benefits of different categories of hybrid-electric and full-electric buses. Few works have so far dealt with extending or reformulating the SDVSP or the MDVSP with explicit consideration of the additional needs stemming from the inclusion of charging/recharging constraints, either from the modelling perspective (12) or from the algorithmic solution development perspective (13-15). These approaches showcase how proper fleet management, under the assumption of homogeneous electric fleet, yields reduced operational costs for the PT operator while, at the same time, reducing overall pollutant emissions.

Most recently, in (16), the authors discuss in details the implications of introducing electrification in the operational planning process for transit systems. Among others, they propose an extension to the MDVSP to consider a homogeneous fleet of electric vehicles operating in a network with multiple charging points, which guarantees that all trips from/to different depots in the network pass through a sufficient amount of charging points, where e-buses will be fully charged. In a different model they also consider a fleet requiring overnight charging only.

To the authors' knowledge, explicitly modelling PT operations consisting of a mixed fleet of full-electric buses - requiring within-day charging at a capacitated infrastructure - and either conventional or plug-in hybrid buses - for which instead no operational charging constraint is present - has so far been neglected. We believe this to be a considerable gap in literature: the shift from internal combustion fleets towards more sustainable alternatives will certainly be gradual in nature, and optimal management in mixed fleet conditions might yield considerable reductions in operational costs, whereas a less-than-careful approach as will be shown later in the results section - could be detrimental to the operator's performance.

When considering online responsiveness to disturbances such as delays, bunching etc., dynamic fleet management approaches in literature focus on optimally allocating deadheading trips $(17,18)$, in order to achieve better reliability. Real-time reaction / prediction in terms of scheduling is however virtually unheard of, at least in transit literature, and has been recognized as a very challenging problem in fields such as city logistics (19).

In the private transport literature, rejection of real-time disturbances is often achieved by means of Model Predictive Control (MPC) (20-23). Employing predictive modelling allows, in principle, to optimally redistribute the available fleet in real-time, without resorting to deadheading additional rolling stock from other terminals or depots. In this work, we will assess whether such a framework can be successfully applied to the instance of dynamic fleet management for transit operations. 


\section{METHODOLOGY}

We begin this section by quickly introducing the main idea behind Model Predictive Control, motivating this framework's choice for dynamic mixed fleet management, and framing the contribution within our chosen case study. We then introduce the developed prediction model, together with the assumptions and modifications required to solve it efficiently, and finally introduce the underlying bus simulation approach, which we employ as "plant model", to represent reality.

\section{Model Predictive Control for dynamic fleet management}

MPC has been successfully employed as a control technique for non-linear systems for the last two decades, ranging in applications from early industrial processes (chemical plants, manufacturing) to vehicular technology and transportation. The rationale behind this success is that the MPC framework (24) allows to apply standard optimisation techniques based upon a white-box prediction model in order to determine the best possible control action, while at the same time retaining the desirable properties of feedback control, in terms of real-time responsiveness and rejection of disturbances. The standard MPC framework, summarised in Figure 1, entails three main components: a prediction model, which is a sufficiently faithful representation of reality, an optimisation approach, which minimises a chosen objective function leveraging the model itself and, finally, a connection to the system being controlled, in terms of sensing/actuation.

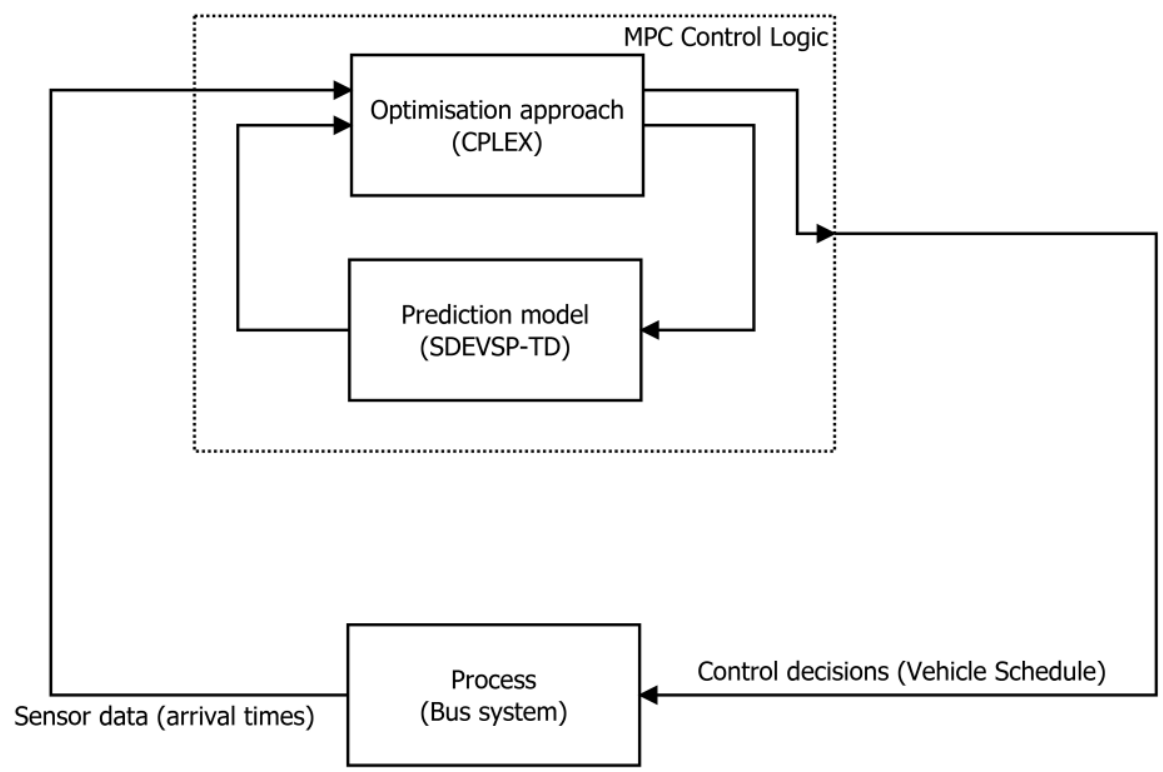

Figure 1: MPC framework description, between parenthesis specific components considered in this work

In the standard Rolling Horizon (RH) approach, the MPC controller operates by, at each time step $t$, optimizing its control decisions subject to the predictions obtained for a certain future duration of time (the prediction horizon, $N_{p}$ ), applying the control decision for the current time $t$ and moving forward to the next time step, where the process is repeated. Depending on the goodness of the chosen prediction model, the length of the prediction horizon and the non-linearity of the underlying phenomenon, the MPC approach might result more or less computationally intensive and more or less successful in controlling the given system.

In this work, we employ as prediction model a Mixed-Integer Linear Programming formulation of the 
vehicle scheduling problem for the specific instance of electric vehicles, subdivided in time windows in order to correctly represent the Rolling Horizon approach. The chosen optimization approach is the branchand-cut approach of IBM's ILOG CPLEX, version 12.7.

\section{The Single Depot Electric Vehicle Scheduling Problem with Time Decomposition (SDEVSP-TD)}

We formulate the problem of scheduling a mixed fleet of electric (e-) and hybrid (h-) buses $I=\{1, \ldots, i\}$ following a set of scheduled trips $J=\{1, \ldots, j\}$, each comprising a desired departure time $D_{j}=\left\{1, \ldots, d_{j}\right\}$ [min], cycle (terminal 1 to terminal 1) duration $T_{j}=\left\{1, . ., t_{j}\right\}$ [min] and total energy required $U_{j}=\left\{1, \ldots, u_{j}\right\}$ $\left[\mathrm{kWh}\right.$. For each trip, decision variables $y_{i, j}^{t}$ and $z_{i, j}^{t}$ describe respectively whether trip $j$ is initiated at time period $t$ by electric or hybrid bus $i$. In order to both allow solving large scale scenarios and correctly represent MPC's Rolling Horizon, we introduce a time window decomposition, so that the full time scale of the problem $T=\{1, \ldots, t\}$ is decomposed in $L_{f}=\left\{l_{1}, \ldots, l_{f}\right\}$ time windows, each bearing its own (possibly heterogeneous) duration. The optimization problem is solved separately and sequentially for each time window, with specific constraints ensuring that correct information flows between the separate problems. This decomposition scheme does however impact optimality: since time lapses are limited in length in order to keep memory requirements at bay, each independent MILP is solved with no knowledge on future trips, whose departures lay beyond the duration of the individual decomposed problem. This implies that decisions taken in time lapse $f$, especially those related to trips departing in its latter time steps, might be sub-optimal from the perspective of the full problem's timetable.

Table 1 introduces the full problem variables' meaning as well as their domain.

Table 1: Problem variables and parameters and their explanation

\begin{tabular}{|l|l|l|}
\hline Var. & Domain & Explanation \\
\hline$y_{i, j}^{t}$ & $\{0,1\}$ & 1 if trip $j$ is initiated by e-bus $i$ at time $t, 0$ otherwise \\
\hline$z_{i, j}^{t}$ & $\{0,1\}$ & 1 if trip $j$ is initiated by h-bus $i$ at time $t, 0$ otherwise \\
\hline$\gamma_{i, j}^{t}$ & $\{0,1\}$ & 1 if e-bus $i$ has sufficient energy $\varepsilon_{i}^{t}$ to perform trip $j$ at time $t, 0$ otherwise \\
\hline$x_{i, m}^{t}$ & $\{0,1\}$ & 1 if e-bus $i$ is being recharged at charging station $m$ at time $t, 0$ otherwise \\
\hline$\varepsilon_{i}^{t}$ & $\leq E, \in \Re^{+}$ & Total energy in kWh that e-bus $i$ has at time $t$ \\
\hline$u_{j}$ & $\Re^{+}$ & Total energy in kWh required to perform trip $j$ \\
\hline$d_{j}$ & $\Re^{+}$ & Preferred departure time for trip $j$ \\
\hline$t_{j}$ & $\Re^{+}$ & Duration of trip $j$ in time steps \\
\hline$s_{i}^{t}$ & $\Re^{+}$ & Slack variable, necessary to ensure that constraint (11) does not violate the domain of $\varepsilon_{i}^{t}$ \\
\hline$E$ & $\Re^{+}$ & Total battery capacity in kWh for all electric buses \\
\hline$A_{i}^{t}$ & $\{0,1\}$ & 1 if e-bus $i$ is not available to perform any trip of the current window problem at time $t, 0$ otherwise \\
\hline$H_{i}^{t}$ & $\{0,1\}$ & 1 if h-bus $i$ is not available to perform any trip of the current window problem at time $t, 0$ otherwise \\
\hline
\end{tabular}

The formulation's objective function, in Eqn. (1), is that of minimizing the total operational cost. 
Rinaldi, Picarelli, Laskaris and Viti

$$
\begin{aligned}
& \min \sum_{t} \sum_{i} \sum_{j} y_{i, j}^{t} \cdot c+r \cdot\left(t-d_{j}\right)+ \\
& \sum_{t} \sum_{i} \sum_{j} z_{i, j}^{t} \cdot \hat{c}+r \cdot\left(t-d_{j}\right)+ \\
& \sum_{m} \sum_{i} \sum_{t} q_{i}^{t} \cdot x_{i, m}^{t} \\
& -\chi \cdot \sum_{t} \sum_{i} \varepsilon_{i}^{t}
\end{aligned}
$$

2 Cost vectors $c$ and $\hat{c}$ are computed as shown in Eqn. (2), considering average cost rates per kWh of energy components $\eta_{1}$ and $\eta_{2}$ for e-buses and h-buses respectively.

$$
\begin{aligned}
& c=\eta_{1} \cdot\left[u_{j}\right] \\
& \hat{c}=\eta_{2} \cdot\left[u_{j}\right]
\end{aligned}
$$

A penalty term $r$ [EUR] is applied to trips being performed later than their preferred departure time, in order to evaluate trade-offs between schedule adherence and operational performance. We consider $M=\{1, \ldots, m\}$ charging stations available at the selected bus terminal, and explicitly take into account the

8 time dependent cost $q_{i}^{t}$ of recharging bus $i$ at time $t$ as part of the operational cost needing minimization.

9 System dynamics are captured by constraints (3-19). The last summation term of Eqn. (1), with $\chi$ chosen

10 appropriately small, is necessary to complement the slack variables introduced in constraint (11).

$$
\sum_{j} y_{i, j}^{t} \leq 1-A_{i}^{t} \forall i, t
$$

$$
\sum_{j} z_{i, j}^{t} \leq 1-H_{i}^{t} \forall i, t
$$

$$
\sum_{t \geq d_{j}} \sum_{i} y_{i, j}^{t}+z_{i, j}^{t}=1 \forall j
$$

$$
\sum_{i} x_{i, m}^{t} \leq 1 \forall m, t
$$

$$
y_{i, j}^{t}-\gamma_{i, j}^{t} \leq 0 \forall i, j, t
$$

$$
\frac{\varepsilon_{i}^{t}}{u_{j}}-\gamma_{i, j}^{t} \geq 0 \forall i, j, t
$$

$$
\frac{\varepsilon_{i}^{t}}{u_{j}}-E \cdot \gamma_{i, j}^{t}<1 \forall i, j, t
$$

$$
E \cdot \sum_{m} x_{i, m}^{t}-\sum_{j} y_{i, j}^{t} \cdot u_{j}+\varepsilon_{i}^{t}-s_{i}^{t}=\varepsilon_{i}^{t+1} \forall i, t
$$

$$
\left\{\begin{array}{l}
\varepsilon_{i, 0}^{0}=E \forall i \\
\varepsilon^{0}{ }_{i, f}=\varepsilon_{i, f-1}^{l_{(f-1)}} \forall i, f
\end{array}\right.
$$

$$
\sum_{j} y_{i, j}^{t}+\frac{s_{i}^{t}}{E} \leq 1 \forall i, t
$$


Rinaldi, Picarelli, Laskaris and Viti

$$
\begin{gathered}
\frac{1}{E} \cdot s_{i}^{t}-\frac{1}{E} \varepsilon_{i}^{t} \leq 0 \forall i, t \\
y_{i, j}^{t}+\sum_{m} x_{i, m}^{t} \leq 1-A_{i}^{t} \forall i, j, t \\
y_{i, j}^{t}+k_{1} \sum_{\bar{t}=t+1}^{t+t_{j}-1} \sum_{\bar{j}} y_{i, \bar{j}}^{\bar{t}} \leq 1 \forall i, t \forall j: t_{j}>1 \\
k_{1}=\frac{1}{t_{j}-1} \\
y_{i, j}^{t}+k_{2} \sum_{\bar{t}=t+1}^{t+t_{j}-1} \sum_{m} x_{i, m}^{\bar{t}} \leq 1 \forall i, t \forall j: t_{j}>1 \\
k_{2}=\frac{1}{|M|} \cdot \frac{1}{t_{j}-1}
\end{gathered}
$$

Constraints (3-4) ensure that no bus (electric or hybrid) can perform more than one trip at a time. Constraint (5) guarantees that all trips must (eventually) be performed by either kind of bus, that trips are not performed more than once, and that trips cannot be performed before their respective preferred departure time.

Constraint (6) implies that an e-bus can be charged at only one charging station at any given time, and, implicitly through the set $M$, that the maximum amount of concurrent recharging buses is limited by the total charging stations available. Constraint (7) ensures that, if an e-bus has no charge left to perform any trip, it will be recharged at a future time. Constraint (8) guarantees that an e-bus will not perform a trip unless it has sufficient energy to do so. Constraints (9-10) describe the characteristics of variable $\gamma_{i, j}^{t}$ in relation to the current available bus energy $\varepsilon_{i}^{t}$ and the required energy to perform trip $u_{j}$. Constraint (11) captures the discharging/recharging dynamics: if an electric bus is performing a trip $j$ at time $t$, its available charge at time $t+1$ will be reduced by $u_{j}$. Conversely, if the electric bus is being recharged at time $t$, its available charge at time $t+1$ will be equal to the total battery capacity $E$. The slack variables $s_{i}^{t}$ absorb any leftover energy $\varepsilon_{i}^{t}$, in order to guarantee that the maximum battery capacity is not violated. Constraint (12) implies that all e-buses begin operations fully charged at the beginning of the simulations, and that between contiguous time windows $f, f+1$ the amount of available energy is conserved for each bus. Constraints (1314) ensure that slack variables can only be utilized during recharging operations. Constraint (15) ensures that an e-bus cannot be recharging and operating simultaneously. Constraints (16-19) ensure that an e-bus performing a trip $j$ whose duration in time steps is greater than one cannot be used to perform any other trip nor recharged until trip $j$ has been completed.

Finally, cross-problem coupling equations (20-23) ensure that the availability of buses whose trips' duration exceeds the length of the current time window $f$ is safeguarded and correctly represented in time window $f+1$. These values are computed once the problem of time window $f$ has been solved, and produce inputs for the problem of time window $f+1$.

$$
\begin{gathered}
\left.A_{i}^{t}\right|_{f}=\left.A_{i}^{t+l_{(f-1)}}\right|_{f-1}+\left.\lambda_{i}^{t}\right|_{f-1} \forall i, f, t \\
\left.H_{i}^{t}\right|_{f}=\left.H_{i}^{t+l_{(f-1)}}\right|_{f-1}+\left.\zeta_{i}^{t}\right|_{f} \forall i, f, t \\
\left.\lambda_{i}^{t}\right|_{f}= \begin{cases}1 & \forall i, t: \exists j, \bar{t}:\left.y_{i, j}^{\bar{t}}\right|_{f}=1 \wedge\left(l_{f}<t \leq \bar{t}+t_{j}\right) \\
0 & \text { otherwise }\end{cases}
\end{gathered}
$$




$$
\left.\zeta_{i}^{t}\right|_{f}= \begin{cases}1 & \forall i, t: \exists j, \bar{t}:\left.z_{i, j}^{\bar{t}}\right|_{f}=1 \wedge\left(l_{f}<t \leq \bar{t}+t_{j}\right) \\ 0 & \text { otherwise }\end{cases}
$$

\section{MATLAB Bus Corridor Simulation software}

The proposed prediction model is linked to a bus simulation model that mimics all the characteristics of real time operation and replicates the different phenomena that may occur at a mesoscopic level. The bus simulation model is coded using Mathworks ${ }^{\mathrm{TM}}$ MATLAB ${ }^{\circledR}$. The inputs of the model are the number of stops with the corresponding demand, per each line, the number of trips and the scheduled riding times between stops. Passenger demand is based on historical data and it is given as input in terms of arrival rates per hour per OD pair. The sources of stochasticity of the model are the dispatching times, the travel times and, consequently, passenger demand. The variability of all three during simulation is modelled by sampling from the probability distribution that best fits with the nature of each variable. By setting the parameters of each distribution, the simulator can be tuned appropriately. Analytically, dispatching times are sampled by Gamma distribution. It is worth mentioning that, for the purposes of this study, only the dispatching times of the trips of the first hour are sampled (based also on the fleet size chosen). For the remainder of the simulation, the dispatching times for the remaining trips are obtained as coupling input from the SDEVSPTD prediction model. An e-bus or h-bus is assigned based on the availability and the cost. Actual riding times are sampled by lognormal distribution, using as average the scheduled riding time and a $20 \%$ standard deviation for the shape and the scale of the distribution. The number of boarding passengers at stops follow Poisson distribution, given the arrival rate and the actual headway at each stop. Since the simulation is stochastic, several replications are conducted with different random seeds.

During simulation, each trip initiates at its dispatching time, which coincides with the arrival at the first stop of the route. For the rest of the stops, the arrival time of the bus is calculated as the sum of the departure time of the vehicle from the previous trip and the actual riding time, as sampled. The difference between the last registered arrival time and the arrival time of the current trip constitutes the actual headway. This is then employed together with the arrival rate at the stop to calculate the number of passengers generated at each stop. The number of boarding passengers is the sum of all passengers originated at the current stop, travelling downstream. The alighting passengers are the passengers on board that were generated upstream and had as destination the current stop. Once the occupancy of the bus is updated, dwell time is calculated as the sum of the products of the boarding and alighting passengers with the corresponding rates as retrieved by (25). After the completion of dwell time, the trip departs from the stop. To appropriately consider the full travel time of each trip as it returns to the terminal from where it was dispatched (in this work we consider circular terminal 1 - terminal 2 - terminal 1 trips), one intermediate stop works as the terminal of the one-way route. At that stop, all remaining passengers are instructed to alight and the trip departs from stop after a predefined time corresponding to its scheduled arrival.

After the completion of each replication, results are generated for the full trajectory of each trip, together with travel and passenger related performance measurements.

\section{EXPERIMENTAL RESULTS}

In this section we first describe the experimental setup, focusing on the chosen case study in the city of Luxembourg. We then showcase two sets of test results: a first set of tests evaluates the impact of additional e-buses in terms of fleet size on two different dynamic management techniques (our proposed model and a simpler Just-In-Time approach), assuming exact information and no disturbances. The second set of tests focuses instead on assessing whether the proposed model is indeed robust when employed as predictor in an MPC framework.

\section{Experimental setup}


As discussed earlier, we consider a real-life case study arising from Luxembourg City, specifically comprising of five urban bus lines, all radiating from the city's central station (Gare Centrale), as highlighted in Figure 2.

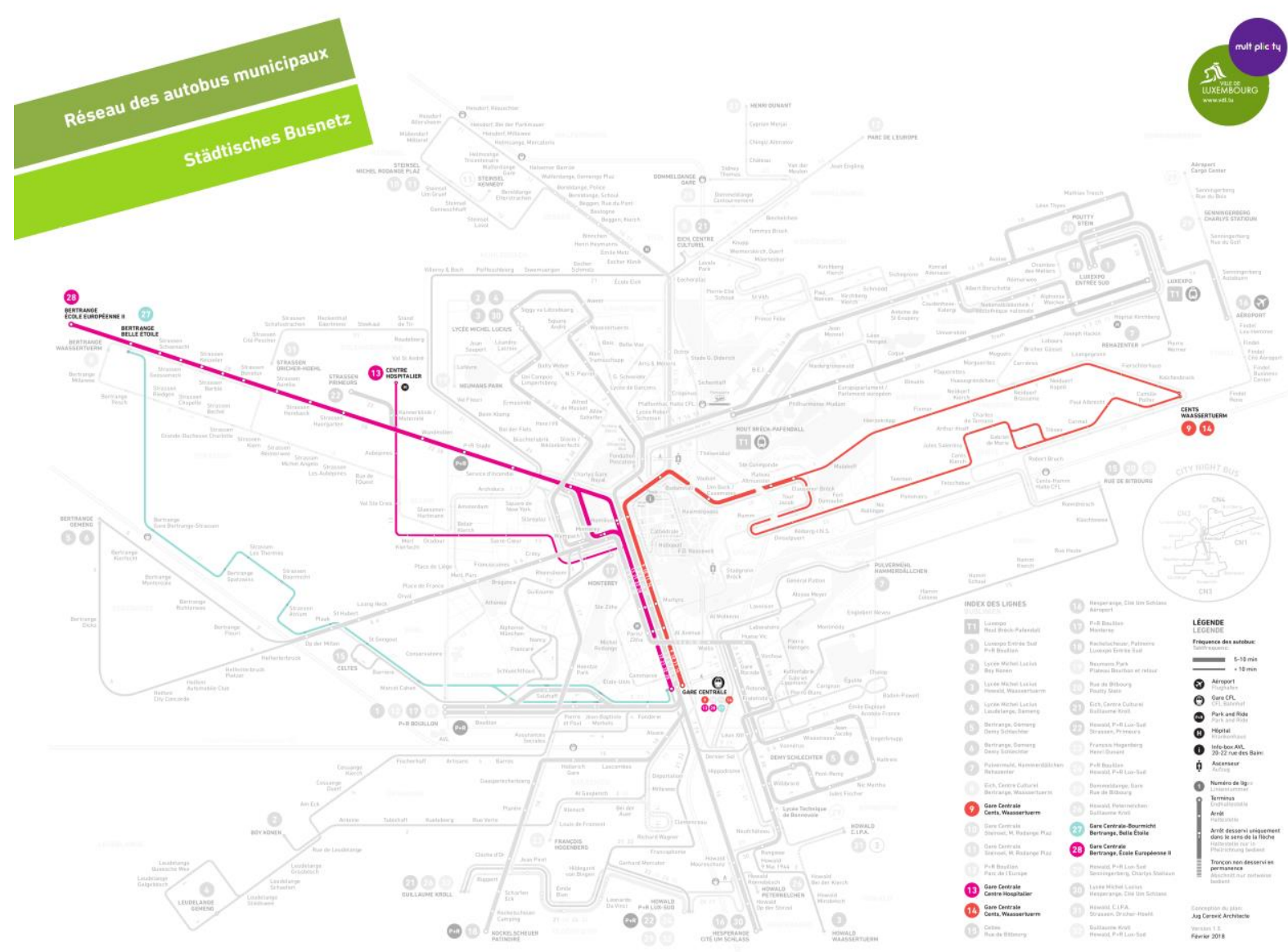

Figure 2: Schematic of Luxembourg City's public transport lines, case study shown in coloring.

Lines 9 and 14 are already being served exclusively through plug-in hybrid buses, with others soon to follow, and two opportunity charging stations have been equipped in the terminal area of Gare Centrale. Starting from this ideal testing ground, we investigate the effect of introducing a mixed fleet including fully electric buses, which will exert a stronger demand on the two charging stations. This allows us to both evaluate whether the existing infrastructure would be sufficient, and to assess the overall impact of electrification in terms of operational costs. We focus on the weekly morning schedule, which consists of 119 trips for these five lines, from $5 \mathrm{am}$ until 1pm, extracted from the openly available GTFS dataset provided within the nation's online data portal (26). The lines operate at an average frequency of about 15 minutes, although some irregularities do arise both within-line and between lines, as shown in Figure 3 (trips have been colored following the same scheme as the schematic in Figure 2). Due to these irregularities, computing the optimal fleet scheduling is considerably challenging. Following our model's formulation, we discretise time considering intervals of 5 minutes. 


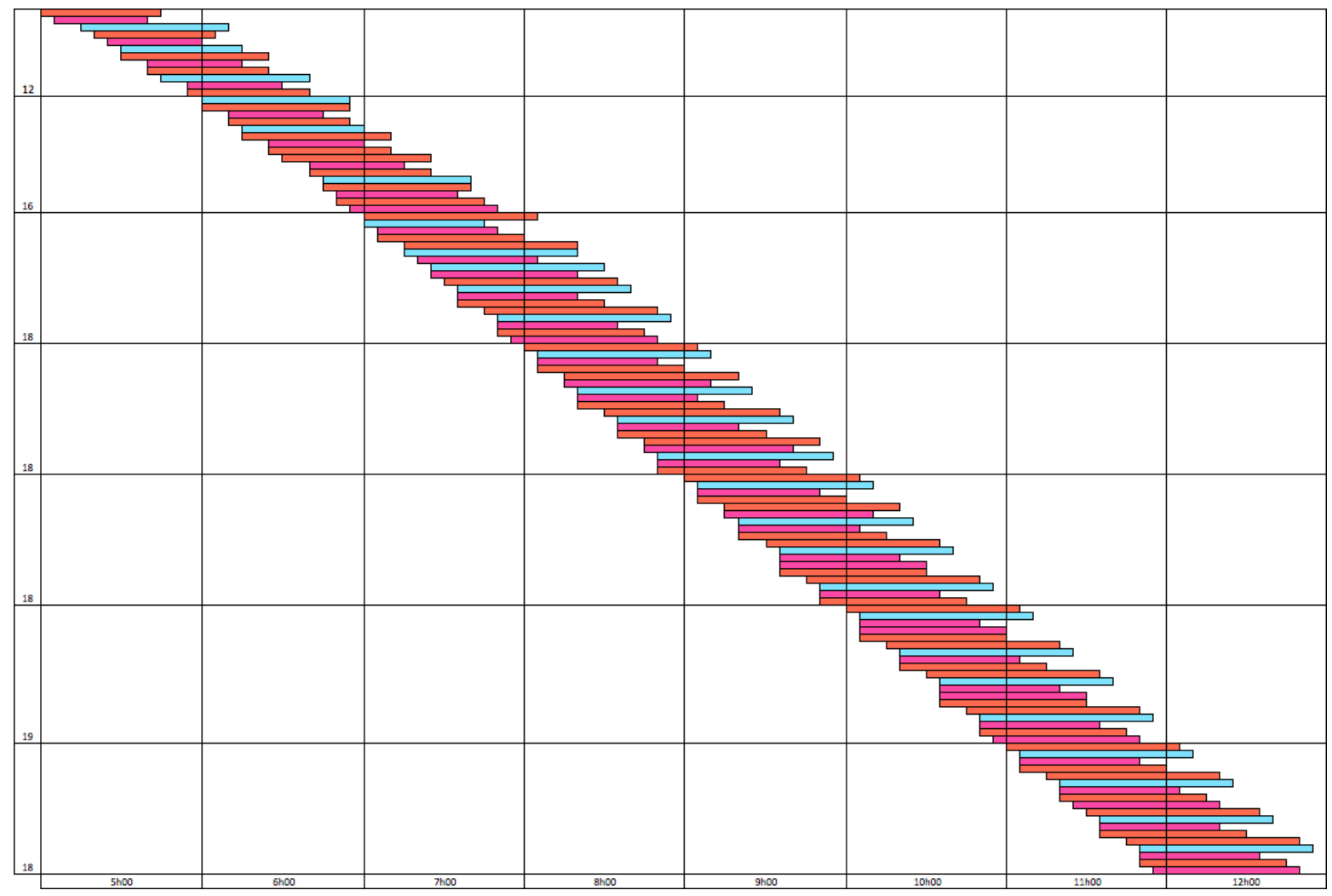

Figure 3: Complete schedule for the five lines considered

In order to faithfully represent reality, we collected average consumption rates for both h-buses and e-buses based both on available literature (10) and on technical sheets provided by Volvo Bus corporation, which supplies the current generation of h-buses to the city of Luxembourg. Specifically, we consider a consumption rate of $1.7 \mathrm{kWh} / \mathrm{km}$ for e-buses and $2.3 \mathrm{kWh} / \mathrm{km}$ for h-buses (including the internal combustion powertrain). In order to monetise these values, we consider for both a generalised cost of 0.27 EUR/kWh, which includes, on top of the cost of electricity itself, an estimation of all costs concerning operational aspects of buses, such as maintenance etc. We assume battery capacity for e-buses to be set at $100 \mathrm{kWh}$, and we apply the current national average price of electricity of $0.15 \mathrm{EUR} / \mathrm{kWh}$ when considering recharging. Even though this assumption is rather conservative compared to peak pricing policies, we will show in what follows that recharging already plays a dominant role in the proposed scenario, and that fleet management should be performed accordingly. For the first set of tests, the possibility of dispatching a trip at a departure time later than the planned value is excluded entirely $(r=\infty)$, whereas a value of $r=5$ EUR is selected for the second, online scenario.

\section{Case Study 1: Fleet size and operational costs}

In this first case study we evaluate the impact of gradually expanding the fleet size allocated to the five lines above, by adding extra e-buses to an otherwise large enough set of h-buses (we suppose availability of h-buses not to be an issue to the feasibility of the problem at hand, in order to isolate the impact of ebuses). We compare the full operational cost in terms of the objective function value (Eqns. 1-2), across three different fleet management techniques: our proposed model (Eqns. 3-19), a Just-In-Time approach and a reference solution obtained employing solely hybrid buses.

We consider a naïve Just-In-Time implementation, which attempts to maximise utilisation of the cheaperto-run electric fleet, disregarding the wider impact that such a policy might have on the overall operational costs in terms of recharging, which instead is used speculatively whenever any e-bus exhibits a charge lower 
than what is necessary.

The Just-In-Time approach operates according to the following simple rules:

- at a given time instant $t$ : if any e-bus $i$ is available and has sufficient charge to perform any trip

- $\quad$ at a given time instant $t$ : if any e-bus $i$ is available but does not have sufficient charge to perform any

For each trip, a scheduling decision is taken by either model in terms of which bus (electric/hybrid) is going to be used to serve it, ensuring that all availability constraints are satisfied.

The results of this first case study are presented in Figure 4.

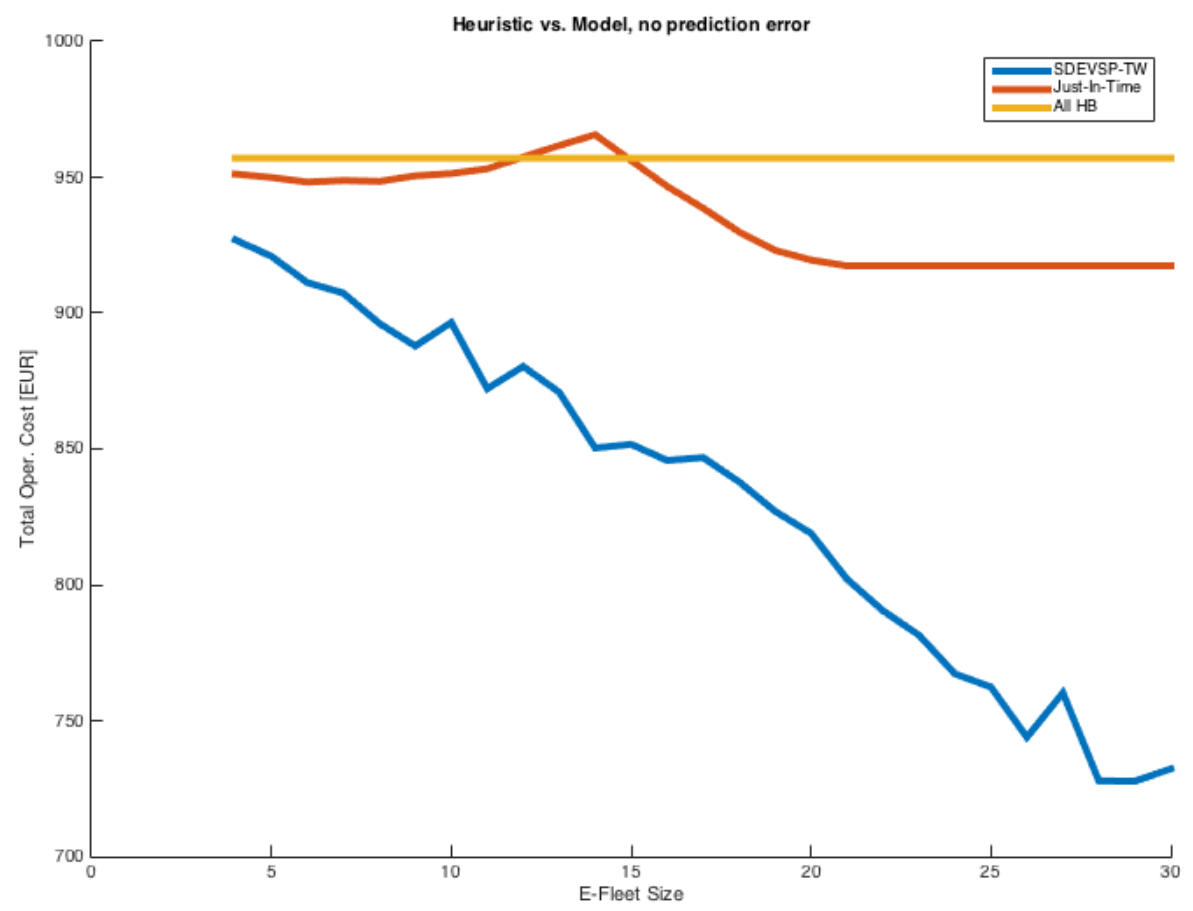

14 Our proposed model performs very well, progressively reducing operational costs as the available e-bus fleet size increases, as is expectable, and reaching substantial savings (ranging from $10 \%$ to over $20 \%$ reduction in total operational cost) when the e-bus fleet size exceeds 14 buses. The naïve just-in-time approach however only achieves smaller operational gains for larger fleet sizes, due to its recharging policy, and is instead worsening overall costs compared to utilising all hybrid buses for fleets ranging between 12 and 14. These results hint at the fact that, indeed, in mixed fleet conditions a myopic approach that tries to maximise the usage of the cheaper running cost alternative is far from ideal, and that instead greater care must be taken in scheduling charging operations.

\section{Case Study 2: dynamic fleet management}

Having verified that our model captures the desired dynamics to a satisfactory level, and that it is indeed capable of yielding substantial gains in terms of objective function under ideal conditions, in this second 
set of tests we investigate whether it can be used in operational conditions, where disturbances such as delays and bunching arise due to variability in demand.

We utilise our proposed model as predictor in an MPC framework, as discussed earlier: at each time step $t$, the approach predicts $N_{p}=30$ minutes into the future, assuming that both dispatching times and trip durations are exactly aligned to the schedule of Figure 3. Once the model reaches optimality, the scheduling decisions for time step $t$ are implemented, both in terms of dispatching time and type of bus utilised (electric/hybrid), and the dispatching information is forwarded to the underlying plant model, which performs the trip(s) accordingly. The exact amount of available rolling stock at time $t$ is obtained in realtime as a derived output from the previous time step's status of the plant model, in terms of trip arrivals.

Passenger demand is simulated along the five lines, in terms of stochastic arrivals, considering a uniform spatial distribution along the lines' stops, and three different levels of total demand (300 pax/h/line, 500 $\mathrm{pax} / \mathrm{h} /$ line, $700 \mathrm{pax} / \mathrm{h} /$ line). As demand increases, the elongated boarding/alighting times cause delays at the line level, with longer lines running the risk of bus bunching under high demand conditions. The actual complete trip durations become known to the prediction model only upon trip completions themselves, meaning that considerable mismatching can occur between the predicted completion times and the realised ones.

We simulate and compare three different fleet management techniques: our proposed model predictive controller, the previously mentioned just-in-time approach and an "oracle" version of our model, computed ex-poste, where exact trip duration and dispatching time information are assumed known at all time steps, providing therefore a "best case" comparison. These results are summarised in Table 2, in terms of percental difference between the realised objective function value and that of an all-hybrid solution.

Table 2: Case Study 2 results. Percental variations compared to an all-hybrid operational cost of 957 EUR.

\begin{tabular}{|l|c|c|c|}
\hline & Just-In-Time & MPC & Oracle \\
\hline Demand: $300 \mathrm{pax} / \mathrm{h} / \mathrm{line}$ & $-1.76 \%$ & $-3.38 \%$ & $-12.71 \%$ \\
\hline Demand: $500 \mathrm{pax} / \mathrm{h} / \mathrm{line}$ & $-0.2 \%$ & $-8.99 \%$ & $-11.84 \%$ \\
\hline Demand: $700 \mathrm{pax} / \mathrm{h} / \mathrm{line}$ & $+1.45 \%$ & $-8.49 \%$ & $-12.77 \%$ \\
\hline
\end{tabular}

As expectable, the "oracle" model performs best in all scenarios, while the chosen just-in-time approach is clearly unsuitable for the given problem. The online MPC approach shows promising results, especially when its reference trip completion times are underestimations compared to the realised ones (that is, for higher demand levels). Conversely, when trips are consistently completed earlier than what is anticipated, the advantage obtained by using a complex prediction model is quickly eroded, reducing from $-12.71 \%$ to $-3.38 \%$. In the "plant" model we assumed that no regularising control (e.g. holding) is active, meaning that in very undersaturated conditions trips can indeed be systematically completed ahead of schedule. In future research we will assess the combined effect of both MPC-based dynamic fleet management and line-wise or system-wise holding strategies. Over all scenarios, the average computational time necessary for CPLEX to optimize the prediction instances of the SDEVSP-TD model was 11.2 seconds, with a standard deviation of 2 seconds, well within the ideal requirements for real-time applicability.

\section{CONCLUSIONS}

In this paper we develop an approach for dynamic mixed electric/hybrid bus fleet management, leveraging a Model Predictive Control framework. After validating the prediction model's capabilities in terms of minimising operational costs in a real-life case study, we assess its online performances considering stochastic trip completion times as arising from a mesoscopic bus simulator approach. All tests conducted are based upon a real-life case study situated in the city of Luxembourg. Test results show that, indeed, the 
proposed model is suitable for a dynamic fleet management application based upon model predictive control, retaining a very solid level of performance even when facing considerable disturbances and delays. Future research includes, first and foremost, the extension of the obtained results to a multi-depot scenario, in order to consider larger instances and case studies. Further investigation will also be performed in evaluating the relationship between charging station location / density and peak electricity pricing by grid operators, framing the latter's dynamic pricing schemes in game theoretical terms and focussing on establishing whether a gain can be found by switching from competition to cooperation.

\section{AUTHOR CONTRIBUTION STATEMENT}

The authors confirm contribution to the paper as follows: study conception and design: M. Rinaldi; model(s) formulation and implementation: E. Picarelli, M. Rinaldi, G. Laskaris; analysis and interpretation of results: M. Rinaldi, E. Picarelli. F. Viti; draft manuscript preparation: M. Rinaldi. G. Laskaris, F. Viti. All authors reviewed the results and approved the final version of the manuscript.

\section{ACKNOWLEDGEMENTS}

The authors would like to acknowledge the financial support of the FNR-CORE project eCoBus C16/IS/11349329.

\section{REFERENCES}

1. Ceder A. Public transit planning and operation: Modeling, practice and behavior. CRC press; 2016.

2. Mobilitéit zesummen erreechen - Modu 2.0 - Développement durable et Infrastructures // Luxembourg - Grands dossiers [Internet]. [cited 2018 Jul 16]. Available from: http://www.developpement-durableinfrastructures.public.lu/fr/grands-dossiers/modu2/index.html

3. Rinaldi M, Parisi F, Laskaris G, D'Ariano A, Viti F. Optimal dispatching of electric and hybrid buses subject to scheduling and charging constraints. In: Proceedings of the 21st IEEE International Conference on Intelligent Transportation Systems. Maui, Hawaii, USA; 2018.

4. Ceder A. Urban transit scheduling: framework, review and examples. J Urban Plan Dev. 2002;128(4):225-244.

5. Desaulniers G, Hickman MD. Chapter 2 Public Transit. In: Barnhart C, Laporte G, editors. Handbooks in Operations Research and Management Science [Internet]. Elsevier; 2007 [cited 2018 Jun 25]. p. 69127. (Transportation; vol. 14). Available from: http://www.sciencedirect.com/science/article/pii/S0927050706140025

6. Ibarra-Rojas OJ, Delgado F, Giesen R, Muñoz JC. Planning, operation, and control of bus transport systems: A literature review. Transp Res Part B Methodol. 2015 Jul 1;77:38-75.

7. Bielli M, Bielli A, Rossi R. Trends in Models and Algorithms for Fleet Management. Procedia - Soc Behav Sci. 2011 Jan 1;20:4-18.

8. Kühne R. Electric buses-An energy efficient urban transportation means. Energy. 2010;35(12):45104513.

9. Li J-Q. Battery-electric transit bus developments and operations: A review. Int J Sustain Transp. 2016;10(3):157-169.

10. Lajunen A. Energy consumption and cost-benefit analysis of hybrid and electric city buses. Transp Res Part C Emerg Technol. 2014 Jan 1;38:1-15.

11. Mahmoud M, Garnett R, Ferguson M, Kanaroglou P. Electric buses: A review of alternative powertrains. Renew Sustain Energy Rev. 2016;62:673-684.

12. Fusco G, Alessandrini A, Colombaroni C, Valentini MP. A model for transit design with choice of electric charging system. Procedia-Soc Behav Sci. 2013;87:234-249.

13. Li J-Q. Transit bus scheduling with limited energy. Transp Sci. 2013;48(4):521-539.

14. Baita F, Pesenti R, Ukovich W, Favaretto D. A comparison of different solution approaches to the vehicle scheduling problem in a practical case. Comput Oper Res. 2000 Nov 1;27(13):1249-69.

15. Wen M, Linde E, Ropke S, Mirchandani P, Larsen A. An adaptive large neighborhood search heuristic 

for the Electric Vehicle Scheduling Problem. Comput Oper Res. 2016 Dec 1;76:73-83.

16. Hall CH, Ceder A, Ekstrom J, Quttineh N-H. Adjustments of Public Transit Operations Planning Process for the Use of Electric Buses. In 2017. p. 20.

17. Eberlein XJ, Wilson NHM, Barnhart C, Bernstein D. The real-time deadheading problem in transit operations control. Transp Res Part B Methodol. 1998 Feb 1;32(2):77-100.

18. Yu B, Yang Z, Li S. Real-time partway deadheading strategy based on transit service reliability assessment. Transp Res Part Policy Pract. 2012 Oct 1;46(8):1265-79.

19. Gendreau M, Potvin J-Y. Issues in Real-Time Fleet Management. Transp Sci. 2004 Nov 1;38(4):3978.

20. Frejo JRD, Camacho EF. Global Versus Local MPC Algorithms in Freeway Traffic Control With Ramp Metering and Variable Speed Limits. IEEE Trans Intell Transp Syst. 2012;13(4):1556-65.

21. Han Y, Yuan Y, Hegyi A, Hoogendoorn SP. Linear Quadratic MPC for Integrated Route Guidance and Ramp Metering. In: 2015 IEEE 18th International Conference on Intelligent Transportation Systems. 2015. p. 1150-5.

22. Rinaldi M, Himpe W, Tampère C. A sensitivity based approach for adaptive decomposition of anticipatory network traffic control. Transp Res C Emerg Technol. 2016 Feb 1;

23. Van De Weg SG, Keyvan-Ekbatani M, Hegyi A, Hoogendoorn SP. Urban Network Throughput Optimization via Model Predictive Control Using the Link Transmission Model. In 2016 [cited 2018 Feb 5]. Available from: https://trid.trb.org/view/1392552

24. Camacho EF, Alba CB. Model Predictive Control. Springer Science \& Business Media; 2013. 423 p.

25. Dueker K, Kimpel T, Strathman J, Callas S. Determinants of Bus Dwell Time. J Public Transp [Internet]. 2004 Mar 1;7(1). Available from: http://scholarcommons.usf.edu/jpt/vol7/iss1/2

26. Luxembourg Open Data Portal [Internet]. [cited 2017 Oct 7]. Available from: https://data.public.lu/en/ 